\title{
Illuminating the genome-wide activity of genome editors for safe and effective therapeutics
}

\author{
Yong Cheng ${ }^{*}$ and Shengdar Q. Tsai ${ }^{*}$ (D)
}

\begin{abstract}
Genome editing holds remarkable promise to transform human medicine as new therapies that can directly address the genetic causes of disease. However, concerns remain about possible undesired biological consequences of genome editors, particularly the introduction of unintended 'off-target' mutations. Here, we discuss both important considerations for therapeutic genome editing and our understanding of the functional impact of undesired off-target mutations. An important challenge for the future will be the development of new approaches for predicting and defining the probable function of unintended genome-editing mutations, which will inspire confidence in the next generation of promising genome-editing therapies.
\end{abstract}

\section{Introduction}

Genome editing is a transformative, broadly applicable technology for making targeted DNA modifications in the genomes of living cells with promise to unlock fundamentally new treatments for human genetic diseases. For example, clinical trials have been initiated to test the safety of a genome-editing approach that has the potential to cure HIV by disruption of CCR5, a gene encoding a co-receptor for HIV. Other promising therapeutic genome-editing approaches include the engineering of better $\mathrm{T}$ cells for cancer immunotherapy [1] or the editing of human hematopoietic stem and progenitor cells (HSPCs) for the treatment of hemoglobinopathies such as sickle cell disease or beta-thalassemia [2].

Current genome editors function either by cutting the DNA itself (nucleases) or by inducing point mutations

* Correspondence: yong.cheng@stjude.org; shengdar.tsai@stjude.org Department of Hematology, St. Jude Children's Research Hospital, 262 Danny Thomas Place, Memphis, TN 38105, USA through the recruitment of natural or engineered deaminases (base editors). There are four major classes of genome-editing nucleases: meganucleases [3], zinc-finger nucleases (ZFNs) [4], transcription activator effector-like nucleases (TALENs) [5], and clustered regularly interspaced palindromic repeats (CRISPR)-Cas RNA-guided nucleases [6]. Nuclease-induced DNA double-strand breaks (DSBs) can be repaired by the endogenous cellular DNA repair machinery, which has a bias towards insertion/deletion (indel) mutations associated with error-prone non-homologous end-joining (NHEJ) over precise homology-directed repair. Base editors are engineered enzymes that are a fusion of a CRISPR-Cas enzyme (used for its DNA-binding properties), a deaminase and, if applicable, a uracil-glycosylase inhibitor. In general, all genome-editing enzymes function by inducing targeted DNA damage that can be converted into useful mutations by the cells own DNA damage repair pathways.

Like many biological enzymes, genome editors do not possess perfect specificity for their targets and as a result may introduce unintended 'off-target' mutations into the genome. Off-target mutagenesis has been observed for all classes of genome editors used to date: meganucleases, ZFNs, TALENs, CRISPR-Cas nucleases, and base editors. However, by careful design, deployment of strategies to improve specificity [7-14], and analysis of genome-wide activity (previously reviewed by Tsai and Joung [15]) in many cases it is possible to avoid introducing detectable levels of off-target mutagenesis.

\section{Safety considerations for therapeutic genome editing}

It is important to see the big picture and fully understand the consequences of harnessing powerful genome-editing enzymes to modify the genomic DNA of living cells. Unintended adverse consequences of therapeutic genome editing could jeopardize warm public support for this entire class of promising new therapies.

(c) The Author(s). 2018 Open Access This article is distributed under the terms of the Creative Commons Attribution 4.0 International License (http://creativecommons.org/licenses/by/4.0/), which permits unrestricted use, distribution, and 
For example, inadvertent activation of proto-oncogenes could predispose patients to cancer, a possible adverse outcome similar to that observed with the use of $\gamma$-retroviral vectors used in gene therapy for several inherited immunodeficiencies [16]. Alternatively, delivery agents or even the genome editors themselves could induce a cellular or immune response. In this Opinion, we focus on the direct intended and unintended consequences of the catalytic activity of genome editors.

There is no 'magic number' or absolute frequency of off-target mutations above which genome editors will be safe or below which they are not. One threshold that has been suggested is the background mutation rate in dividing cells, which has been estimated to be $\sim 1.6 \times 10^{-8}$ [17]. This type of arbitrary threshold is irrelevant, however, because genome-editing activity is systematic and not random, so mutations at an off-target site that could inactivate a tumor-suppressor (such as P53) should be considered dangerous even at frequencies well below the background mutation rate, whereas high-frequency mutations in an inert non-coding region might be completely harmless.

Safety of therapeutic genome-editing approaches should be evaluated with a nuanced risk-benefit analysis. The obvious and greatest risk is unintended mutagenesis that confers cells with a proliferative advantage that leads to clonal expansion and malignant cellular transformation. In some cases, however, pro-proliferative mutations may serve to enhance the efficacy of treatment [18]. Some unintended effects may dampen the efficacy of a therapeutic strategy but may not be inherently dangerous. Pre-existing immunity to genome editors may result in the rapid clearance of edited cells, or an innate immune response to editing components could lead to cellular toxicity. The number and nature of cells that are exposed to genome editors is another risk modifier. Hundreds of millions of cells would typically be edited in ex vivo genome editing of human HSPCs or $T$ cells, whereas in vivo editing of the liver could affect billions of cells. The greater the number of cells that are modified, the greater the possibility that one of them may accumulate undesired oncogenic driver mutations. Primary cells that have limited replicative potential may have a lower risk of transformation, whereas a deleterious mutation to a self-renewing stem cell may have long-term adverse consequences.

The potential benefits of genome-editing strategies may be more easily understood. A few notable examples include: 1) human T cells can be edited to disrupt CCR5 and confer resistance to HIV infection [19]; 2) HSPCs from sickle cell disease patients can be modified to induce the expression of fetal hemoglobin as a functional replacement for defective adult hemoglobin in differentiated red blood cell progeny [2]; and 3) human T cells with enhanced tumor rejection properties can be engineered by targeted insertion of chimeric antigen receptors into the T-cell receptor alpha constant (TRAC) locus [1]. These benefits can be initially assessed through rigorous pre-clinical studies that measure the degree of efficient on-target editing and its functional consequences in cellular and animal models.

\section{State-of-the-art detection and prediction methods: Capabilities and limitations}

Over the years, dramatic progress has been made in developing techniques to experimentally define the genome-wide activity of genome editors. These methods can be broadly divided into two categories: 1) cell-based strategies such as HTGTS (high-throughput, genome-wide translocation sequencing), BLESS/BLISS (breaks labeling, enrichment on streptavidin and sequencing/breaks labeling in situ and sequencing), GUIDE-seq (genome-wide unbiased identification of DSBs enabled by sequencing), and integrase-deficient lentivirus (IDLV) capture [20-25]); and 2) in vitro methods (CIRCLE-seq, Digenome-seq, and SITE-seq [26-29]), which we have previously reviewed in detail $[15,30]$. The cell-based methods have the advantage of being able to detect cell-specific genome editing activity directly but have limitations in their sensitivity. In vitro methods are generally more sensitive and more comprehensive than cell-based methods, but characteristic nuclease-induced indel mutations cannot always be detected at all cleavage sites because of cell-specific chromatin accessibility, competition from endogenous cellular DNA-protein binding, or the concentration of genome-editing proteins that is achievable in cells.

Presently, the development of accurate and comprehensive computational or in silico methods for predicting genome-wide off-target activity is limited by the availability of large-scale training and validation datasets. Experimentally, off-target sites have been identified with up to six mismatches relative to their intended target site for CRISPR-Cas nucleases [20], up to eight mismatches for ZFNs [31], and up to 12 mismatches for TALENs [32]. Accurate in silico prediction of off-target activity is extremely difficult because the search space for potential off-targets is very large while the number of true off-targets is relatively small. Currently, it is possible to exclude particularly poor on-target sites that have closely related off-target sites by using computational tools such as Cas-OFFinder [33]. In the future, the generation of large-scale genome-wide genome-editing activity datasets, coupled with the development of machine-learning methods, may enable further progress in this challenging area. Until such in silico prediction methods mature and have been carefully vetted and prospectively validated, sensitive and unbiased experimental 
methods should be prioritized over in silico methods for defining the genome-wide activity of genome editors, because such experimental methods can sensitively and accurately identify sites without limiting pre-defined assumptions.

We should remain keenly aware of both the capabilities and limitations of the experimental methods that have been developed for discovering the genome-wide activity of genome editors. A common blind spot for both discovery and validation methods is their reliance on short-read high-throughput sequencing. Nearly 50\% of the human genome is composed of repetitive elements [34], and so many regions remain difficult to uniquely map and are inaccessible to modern short-read, high-throughput sequencing methods [35]. Although they are difficult to sequence and map, repetitive elements are important as they often play an important role in tissue-specific gene regulation and host transcription-factor binding sites [36, 37]. Methods such as CIRCLE-seq that can identify full off-target sites in a sequencing read pair can overcome this mapping limitation, as they can be run in a reference genome-independent mode [26]. For validation, the error-rate of the high-throughput sequencing process, typically around $0.1 \%$, can be limiting because it obscures mutational activity below this threshold. Two reports that found large deletions that were induced by CRISPR-Cas nucleases reinforce the point that our ability to detect genome-editing mutations is highly dependent on the method of observation [38, 39]. Using short-read, high-throughput sequencing technologies, large deletions, inversions, or structural rearrangements can easily be missed. Nuclease-induced DSBs can also interact with randomly occurring DSBs to generate chromosomal translocations [21].

Complementary methods should be used as required to obtain the broadest possible view of the activity of genome editors. When feasible in the cell types being studied, the pairing of cell-based methods such as GUIDE-seq with in vitro genome-wide activity profiling methods such as CIRCLE-seq or Digenome-seq may provide more information than either method alone. For validation of on-target and off-target activity, unidirectional anchored sequencing methods such as amplicon sequencing (AMP-seq) [40] and UDiTaS [41] may reveal information about structural rearrangements that cannot be observed using standard bidirectional PCR. Cytogenetic or other methods for visualizing large-scale genomic rearrangements may also play an important role in understanding the full impact of genome editing, revealing aspects that cannot be appreciated through the use of genomic sequencing methods alone. These methods may be especially important for genome-editing applications such as $\mathrm{T}$ cell-based cancer immunotherapy strategies where multiplex genome editing is often desirable (for example, to insert a chimeric antigen receptor and to knock out genes associated with $\mathrm{T}$-cell exhaustion simultaneously).

Cell-based surrogate assays (where the cells used to analyze specificity do not match the target cell type) should be avoided because they do not account for genetic or epigenetic differences between the surrogate and the target cell type. There may be differences in epigenetic factors or chromatin organization between the surrogate and target cells. In certain challenging primary cell types such as human hematopoietic stem cells (HSCs), where it is difficult to use assays such as GUIDE-seq, a combination of in vitro discovery and targeted validation is preferable.

Genome-wide assays to define genome-editing activity should be designed to read out the enzymatic activity of interest as directly as possible. In widely used Streptococcus pyogenes Cas9, DNA cleavage is allosterically regulated by extensive RNA-DNA complementarity beyond that required for binding [42, 43]. Therefore, assays such as chromatin immunoprecipitation sequencing (ChIP-seq) that can be used to measure the binding of catalytically inactive or dead Cas9 (dCas9) are not generally predictive of genuine Cas9 cleavage sites [42]. Similarly, nuclease-induced mutagenesis is not necessarily correlated with base editing, which depends largely on the DNA-binding and helicase activities of Cas9 [44]. These examples illustrate why assays that are designed to read out the catalytic or mutational activity of the genome editors themselves are crucial and likely to be more informative than other studies.

An eyes-wide-open approach to defining the fundamental genome-wide activity of genome editors should inspire not diminish confidence in their safety. Increased assay sensitivity does not imply that all genome editors are flawed but should be considered as the means for rational data-driven selection of editors that are truly the best choice for each clinical application. For example, these highly sensitive state-of-the-art methods enable rigorous examination of the relative merits of engineered variants or newly discovered genome editors.

\section{A framework for predicting functional mutation sites}

Risks associated with genome editing should be considered as an integrated measure of the location, frequency, and functional impact of the resulting off-target activity. Significant advances have been made through the development of sophisticated genome-wide methods to determine the location and frequency of unintended off-target activity, but discerning functional impact remains a major challenge. Our understanding of the genome-wide activity of genome editors remains 
superficial, like an incomplete nautical map showing potential hazards without indicating how dangerous they might be. To chart a safe course towards genome-editing therapeutics, it will be important to develop new methods that will allow us to see below the surface and functionally understand the consequences of genome-editing activity (see Fig. 1). The question is: how can we distinguish harmful from benign sites of off-target mutations? Here, we discuss how off-target mutations may affect normal genome functions and propose criteria for the design of therapeutic genome editing.

Obviously, off-target sites that are located within protein-coding sequences are most likely to have a functional impact and should be avoided if possible. Small indels are the most common type of mutations introduced by genome-editing nucleases, and these mutations can lead to the frameshift of protein-coding sequences and functional gene knockout. Indels that are close to the $5^{\prime}$ end of transcripts start sites or within functional domains would be predicted to cause more severe side effects. Although avoidance of off-target mutations within protein-coding sequences is preferred, there may be exceptions to this rule. Not all genes are actively expressed in a given cell type, so genome editors that induce off-target mutations that are located within silenced genes may still be considered, especially if no better alternatives are available. In some cases, off-target mutations in protein-coding sequences that are closely related to the target sequence may be unavoidable but acceptable because they are benign. As protein-coding sequences contribute to less than $2 \%$ of the human genome, we anticipate the vast majority of off-target mutations will be found in non-coding DNA sequences. To date, our knowledge of the function and organization of non-coding sequences remains elusive, further increasing the difficulty of accurately predicting the functional consequences of mutations at non-coding off-target sites.

Although there is still no gold standard for categorizing deleterious non-coding mutations, we can outline some foundational principles for assessing off-target activity in these regions (see Fig. 2). First, epigenetic signals such as histone modifications (H3K27ac, H3K4me1, and H3K4me3), chromatin openness, and transcription factor occupancy have been widely used as markers for active regulatory DNA sequences [45-47], and genome editors that induce off-target mutations overlapping these features should be avoided. Second, DNA sequences that are under strong purifying selection or positive selection are likely to be associated with important biological functions and should not be modified either. Evolutionarily constrained regions of the human genome are highly enriched in pathogenic variants and new maps of these sequence constraints from thousands of people may help to infer the locations of important non-coding genetic elements [48]. Third, because the functions of non-coding sequences are highly tissue- or cell type-specific, the evaluation of non-coding mutation effects should be conducted in the context of the edited cell type. The human genome is spatially organized into

(a)

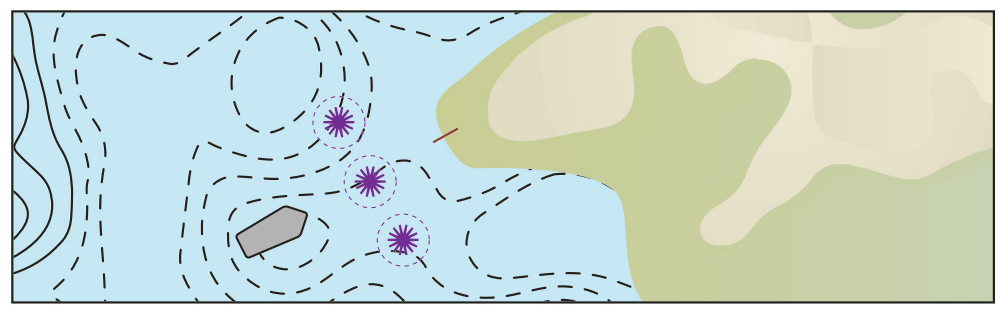

(b)

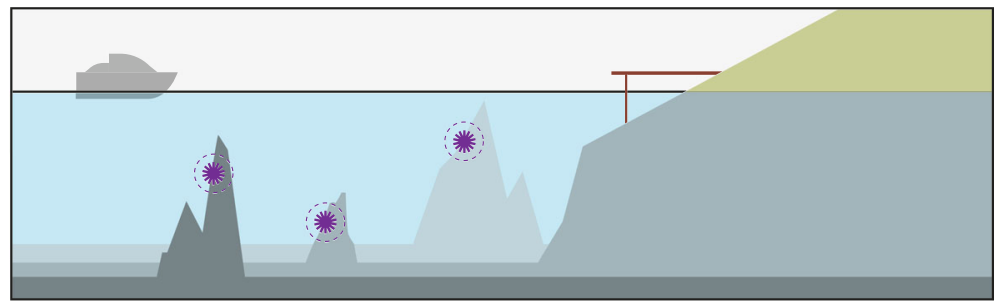

Fig. 1 Charting a course towards safe genome editing. a Like an incomplete nautical map, current methods for defining the genome-wide activities of genome editors identify the locations of potential hazards without offering additional insight into the level of functional risk. $\mathbf{b}$ Methods that look below the surface will allow us to understand the level of risk associated with particular hazards and will help to increase confidence in genome-editing strategies 


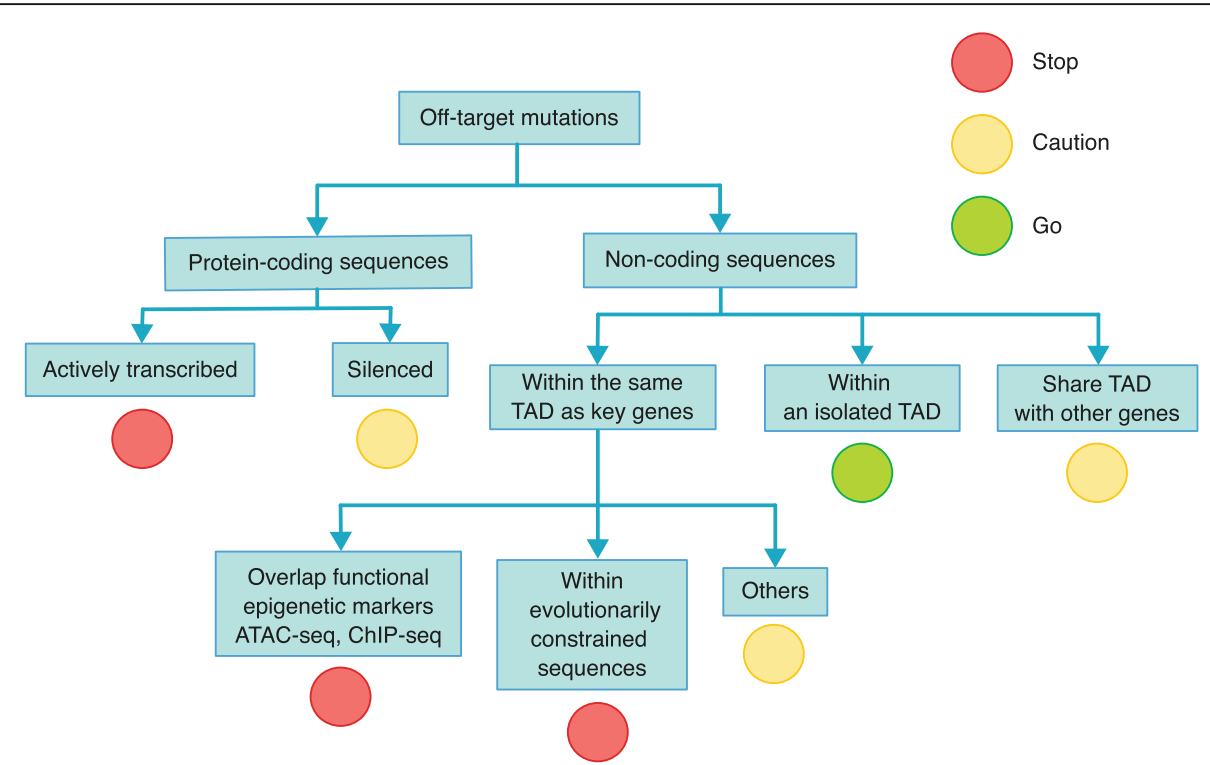

Fig. 2 Functional classification of sites of off-target genome-editing mutations. A proposed framework outlining principles for defining the function of sites of off-target mutation. Some sites should be strictly avoided (red), others may require caution in interpretation (yellow), and a few in isolated topologically associated domains (TADs) may be non-functional and unlikely to be deleterious (green)

different units called topologically associating domains (TADs). Most interactions between regulatory sequences and target genes occur within the same TAD $[49,50]$. Thus, the prediction of non-coding mutations needs to be conducted in the context of TAD structure.

Nevertheless, an approach of avoiding all genomic loci that overlap with DNA sequences enriched with epigenetic signals may be overly cautious as there is no guarantee that all such sequences will have regulatory functions. Although the impact of indels on coding sequences could be highly disruptive, small indels may not be sufficient to disrupt the functions of many non-coding regulatory sequences [51] and the natural occurrence of polymorphisms within those loci among the healthy population might suggest that a considerable percentage of those mutations are actually functionally neutral [52].

\section{Present and future outlook for genome-editing therapeutics}

Some published reports have been interpreted as indicating a need for concern about the prospects of certain therapeutic genome-editing technologies. Schaefer et al. [53] initially claimed that Cas9 induces genome-wide point mutations and two groups reported that CRISPR-Cas9-mediated DSBs activate a TP53 response that had to be suppressed before they could achieve efficient genome editing in certain cell types $[54,55]$. In our view, therapeutic genome editing should continue to be approached rigorously and carefully, but there is no overt cause for alarm.
The Schaefer et al. [53] report, which has subsequently been retracted, claimed that CRISPR-Cas nucleases induce high-frequency point mutations genome-wide. Instead, because the genetic relationship between the edited and control mice remains unclear, the simplest explanation for the genetic differences observed is pre-existing heterogeneity in the genetic background of the mice that were involved in this study [56-60]. Careful follow-up studies employing trio sequencing of genome-edited mice found no evidence of unexpected Cas9-induced point mutations at levels above background [61].

Most currently envisioned clinical genome-editing strategies do not depend on TP53 inhibition or genetic selection of modified cells. In many cases, clinical strategies plan to edit large numbers of primary cells such as HSPCs or T cells ex vivo for later direct reinfusion into the patient. As genetic selection for correctly modified cells is typically not feasible and not performed, there is no increased risk of enriching for cells that have previously acquired TP53 mutations. Nevertheless, there is an exception in situations where the edited cells have a strong selective advantage over unedited cells. An example of this is the gene correction of IL2RG for $\mathrm{X}$-linked severe combined immunodeficiency (SCID-X1), where IL2RG-corrected $\mathrm{B}$ and $\mathrm{T}$ cells have a strong advantage over IL2RG mutant cells in repopulating the thymus. In these special cases, it may be important to achieve high editing efficiency in a number of cells that is sufficient to minimize the possibility of selectively expanding cell clones harboring unwanted tumorigenic mutations [62-64]. 
For clinical genome editing, it may be important to account for genetic variation between individuals, but the impact of this variation will need to be ascertained experimentally. Certainly, all practitioners will take into account and typically avoid on-target sites in which there is common genetic variation. There are clear reports of sites at which individual single nucleotide variants can affect the activity of genome editors [26, 65], but the general impact of human genetic variation on genome-wide activity is less clear. Understanding these effects will require the development of scalable, high-throughput versions of sensitive and unbiased genome-scale methods to define the genome-wide activity of genome editors. With better tools, we anticipate that it may become routine to check the genome-wide activity of editors in the context of an individual's specific genomic DNA.

Over the past several years, remarkable progress has been made not only in fundamental genome-editing technologies but also in the tools used to illuminate their genome-wide editing activity. These methods serve the important purpose of highlighting locations of unintended mutagenesis and have enabled the careful selection of clinical genome-editing strategies and targets that are now progressing through human clinical trials. Although we can now see the unintended mutagenic activity of genome editors in living cell genomes much more clearly, an important future challenge will be to develop new ways to interpret the functional biological consequences of this activity. Advances in our capability to illuminate and interpret global genome-editing activity will inspire confidence in the safety of the next generation of promising genome-editing therapies.

\section{Abbreviations \\ CRISPR: Clustered regularly interspaced palindromic repeats; DSB: Double- strand break; HSPC: Hematopoietic stem and progenitor cell; indel: Insertion/ deletion; TAD: Topologically associated domain; TALEN: Transcription activator effector-like nuclease; ZFN: Zinc-finger nuclease \\ Funding \\ This work was supported by St. Jude Children's Research Hospital and ALSAC, St. Jude Children's Research Hospital Collaborative Research Consortium on Novel Gene Therapies for Sickle Cell Disease, the Doris Duke Charitable Foundation (2017093), and a National Institutes of Health (NIH) grant U01HL145793 (to SQT).}

\section{Authors' contributions}

YC and SQT wrote the manuscript. Both authors read and approved the final manuscript.

\section{Ethics approval}

Not applicable for an Opinion article.

\section{Competing interests}

SQT has financial interests in Monitor Biotechnologies. SQT is a co-inventor on patents describing the GUIDE-seq and CIRCLE-seq methods that have been licensed to Monitor Biotechnologies. YC declares that they have no competing interests.

\section{Publisher's Note}

Springer Nature remains neutral with regard to jurisdictional claims in published maps and institutional affiliations.

Published online: 22 December 2018

\section{References}

1. Eyquem J, Mansilla-Soto J, Giavridis T, van der Stegen SJ, Hamieh M, Cunanan KM, et al. Targeting a CAR to the TRAC locus with CRISPR/Cas9 enhances tumour rejection. Nature. 2017;543:113-7.

2. Traxler EA, Yao Y, Wang Y-D, Woodard KJ, Kurita R, Nakamura Y, et al. A genome-editing strategy to treat $\beta$-hemoglobinopathies that recapitulates a mutation associated with a benign genetic condition. Nat Med. 2016;22: 987-90.

3. Silva G, Poirot L, Galetto R, Smith J, Montoya G, Duchateau P, et al. Meganucleases and other tools for targeted genome engineering: perspectives and challenges for gene therapy. Curr Gene Ther. 2011;11:11-27.

4. Urnov FD, Rebar EJ, Holmes MC, Zhang SH, Gregory PD. Genome editing with engineered zinc finger nucleases. Nat Rev Genet. 2010;11:nrg2842.

5. Joung KJ, Sander JD. TALENs: a widely applicable technology for targeted genome editing. Nat Rev Mol Cell Biol. 2012;14:49-55.

6. Sander JD, Joung KJ. CRISPR-Cas systems for editing, regulating and targeting genomes. Nat Biotechnol. 2014;32:347-55.

7. Doyon Y, Vo TD, Mendel MC, Greenberg SG, Wang J, Xia DF, et al. Enhancing zinc-finger-nuclease activity with improved obligate heterodimeric architectures. Nat Methods. 2011:8:74-9.

8. Hubbard BP, Badran AH, Zuris JA, Guilinger JP, Davis $K$, Chen $L$, et al. Continuous directed evolution of DNA-binding proteins to improve TALEN specificity. Nat Methods. 2015;12:939-42.

9. Fu Y, Sander JD, Reyon D, Cascio VM, Joung KJ. Improving CRISPR-Cas nuclease specificity using truncated guide RNAs. Nat Biotechnol. 2014;32: nbt.2808.

10. Ran AF, Hsu PD, Lin C-Y, Gootenberg JS, Konermann S, Trevino AE, et al, Double nicking by RNA-guided CRISPR Cas9 for enhanced genome editing specificity. Cell. 2013;154:1380-9.

11. Tsai SQ, Wyvekens N, Khayter C, Foden JA, Thapar V, Reyon D, et al. Dimeric CRISPR RNA-guided Fokl nucleases for highly specific genome editing. Nat Biotechnol. 2014;32:569-76.

12. Guilinger JP, Thompson DB, Liu DR. Fusion of catalytically inactive Cas9 to Fokl nuclease improves the specificity of genome modification. Nat Biotechnol. 2014;32:nbt.2909.

13. Kleinstiver BP, Pattanayak V, Prew MS, Tsai SQ, Nguyen NT, Zheng Z, et al. High-fidelity CRISPR-Cas9 nucleases with no detectable genome-wide offtarget effects. Nature. 2016;529:490-5.

14. Slaymaker IM, Gao L, Zetsche B, Scott DA, Yan WX, Zhang F. Rationally engineered Cas9 nucleases with improved specificity. Science. 2016;351:84-8.

15. Tsai $Q$, Joung J. Defining and improving the genome-wide specificities of CRISPR-Cas9 nucleases. Nat Rev Genet. 2016:17:300-12.

16. Hacein-Bey-Abina S, Kalle VC, Schmidt M, McCormack M, Wulffraat N, Leboulch $\mathrm{P}$, et al. LMO2-associated clonal T cell proliferation in two patients after gene therapy for SCID-X1. Science. 2003;302:415-9.

17. Vermulst $M$, Bielas $J H$, Loeb LA. Quantification of random mutations in the mitochondrial genome. Methods. 2008;46:263-8.

18. Fraietta JA, Nobles $C L$, Sammons MA, Lundh $S$, Carty SA, Reich $T J$, et al. Disruption of TET2 promotes the therapeutic efficacy of CD19-targeted T cells. Nature. 2018;558:307-12.

19. Tebas P, Stein D, Tang WW, Frank I, Wang SQ, Lee G, et al. Gene editing of CCR5 in autologous CD4 T cells of persons infected with HIV. N Engl J Med. 2014;370:901-10.

20. Tsai SQ, Zheng Z, Nguyen NT, Liebers M, Topkar W, Thapar V, et al. GUIDEseq enables genome-wide profiling of off-target cleavage by CRISPR-Cas nucleases. Nat Biotechnol. 2015;33:187-97.

21. Frock RL, Hu J, Meyers RM, Ho Y-J, Kii E, Alt FW. Genome-wide detection of DNA double-stranded breaks induced by engineered nucleases. Nat Biotechnol. 2015;33:179-86.

22. Crosetto N, Mitra A, Silva M, Bienko M, Dojer N, Wang Q, et al. Nucleotideresolution DNA double-strand break mapping by next-generation sequencing. Nat Methods. 2013;10:361-5.

23. Ran AF, Cong L, Yan WX, Scott DA, Gootenberg JS, Kriz AJ, et al. In vivo genome editing using Staphylococcus aureus Cas9. Nature. 2015:520: nature14299. 
24. Yan WX, Mirzazadeh R, Garnerone S, Scott D, Schneider MW, Kallas T, et al. BLISS is a versatile and quantitative method for genome-wide profiling of DNA double-strand breaks. Nat Commun. 2017;8:15058.

25. Wang X, Wang Y, Wu X, Wang J, Wang Y, Qiu Z, et al. Unbiased detection of off-target cleavage by CRISPR-Cas9 and TALENs using integrase-defective lentiviral vectors. Nat Biotechnol. 2015:33:175-8.

26. Tsai SQ, Nguyen NT, Malagon-Lopez J, Topkar W, Aryee MJ, Joung KJ. CIRCLE-seq: a highly sensitive in vitro screen for genome-wide CRISPR-Cas9 nuclease off-targets. Nat Methods. 2017;14:607-14.

27. Park J, Childs L, Kim D, Hwang G-H, Kim S, Kim S-T, et al. Digenome-seq web tool for profiling CRISPR specificity. Nat Methods. 2017;14:548-9.

28. Cameron P, Fuller CK, Donohoue PD, Jones BN, Thompson MS, Carter MM, et al. Mapping the genomic landscape of CRISPR-Cas9 cleavage. Nat Methods. 2017;14:600-6.

29. Lazzarotto CR, Nguyen NT, Tang X, Malagon-Lopez J, Guo JA, Aryee MJ, et al. Defining CRISPR-Cas9 genome-wide nuclease activities with CIRCLE-seq. Nat Protoc. 2018;13:2615-42.

30. Tsai SQ. Discovering the genome-wide activity of CRISPR-Cas nucleases. ACS Chem Biol. 2018;13:305-8.

31. Sander JD, Ramirez CL, Linder SJ, Pattanayak V, Shoresh N, Ku M, et al. In silico abstraction of zinc finger nuclease cleavage profiles reveals an expanded landscape of off-target sites. Nucleic Acids Res. 2013;41:e181.

32. Guilinger JP, Pattanayak V, Reyon D, Tsai SQ, Sander JD, Joung KJ, et al. Broad specificity profiling of TALENs results in engineered nucleases with improved DNA-cleavage specificity. Nat Methods. 2014;11:429-35.

33. Bae S, Park J, Kim J-S. Cas-OFFinder: a fast and versatile algorithm that searches for potential off-target sites of Cas9 RNA-guided endonucleases. Bioinformatics. 2014:30:1473-5.

34. Treangen TJ, Salzberg SL. Repetitive DNA and next-generation sequencing: computational challenges and solutions. Nat Rev Genet. 2011;13:36-46.

35. Zook JM, Chapman B, Wang J, Mittelman D, Hofmann O, Hide W, et al. Integrating human sequence data sets provides a resource of benchmark SNP and indel genotype calls. Nat Biotechnol. 2014;32: 246-51.

36. Xie M, Hong C, Zhang B, Lowdon RF, Xing X, Li D, et al. DNA hypomethylation within specific transposable element families associates with tissue-specific enhancer landscape. Nat Genet. 2013;45:ng.2649.

37. Sundaram V, Cheng Y, Ma Z, Li D, Xing X, Edge $P$, et al. Widespread contribution of transposable elements to the innovation of gene regulatory networks. Genome Res. 2014;24:1963-76.

38. Kosicki M, Tomberg K, Bradley A. Repair of double-strand breaks induced by CRISPR-Cas9 leads to large deletions and complex rearrangements. Nat Biotechnol. 2018:36:765-71.

39. Adikusuma F, Piltz S, Corbett MA, Turvey M, McColl SR, Helbig KJ, et al. Large deletions induced by Cas9 cleavage. Nature. 2018;560:E8-9.

40. Zheng Z, Liebers M, Zhelyazkova B, Cao Y, Panditi D, Lynch KD, et al. Anchored multiplex PCR for targeted next-generation sequencing. Nat Med. 2014;20:1479-84.

41. Giannoukos G, Ciulla DM, Marco E, Abdulkerim HS, Barrera LA, Bothmer A et al. UDiTaS ${ }^{\top M}$, a genome editing detection method for indels and genome rearrangements. BMC Genomics. 2018;19:212.

42. Wu X, Scott DA, Kriz AJ, Chiu AC, Hsu PD, Dadon DB, et al. Genome-wide binding of the CRISPR endonuclease Cas 9 in mammalian cells. Nat Biotechnol. 2014;32:670-6.

43. Sternberg SH, LaFrance B, Kaplan M, Doudna JA. Conformational control of DNA target cleavage by CRISPR-Cas9. Nature. 2015;527:110-3.

44. Kim D, Lim K, Kim S-T, Yoon S, Kim K, Ryu S-M, et al. Genome-wide target specificities of CRISPR RNA-guided programmable deaminases. Nat Biotechnol. 2017:35:nbt.3852

45. Heintzman ND, Hon GC, Hawkins DR, Kheradpour P, Stark A, Harp LF, et al. Histone modifications at human enhancers reflect global cell-type-specific gene expression. Nature. 2009;459:108-12

46. Creyghton MP, Cheng AW, Welstead GG, Kooistra T, Carey BW, Steine EJ, et al. Histone H3K27ac separates active from poised enhancers and predicts developmental state. Proc Natl Acad Sci U S A. 2010;107: 21931-6.

47. Heintzman ND, Stuart RK, Hon G, Fu Y, Ching CW, Hawkins DR, et al. Distinct and predictive chromatin signatures of transcriptional promoters and enhancers in the human genome. Nat Genet. 2007;39:311-8.

48. di lulio J, Bartha I, Wong EH, Yu H-C, Lavrenko V, Yang D, et al. The human noncoding genome defined by genetic diversity. Nat Genet. 2018;50:333-7.
49. Nora EP, Lajoie BR, Schulz EG, Giorgetti L, Okamoto I, Servant N, et al. Spatial partitioning of the regulatory landscape of the $\mathrm{X}$-inactivation Centre. Nature. 2012;485:381-5.

50. Dixon JR, Selvaraj S, Yue F, Kim A, Li Y, Shen Y, et al. Topological domains in mammalian genomes identified by analysis of chromatin interactions. Nature. 2012:485:376-80

51. Canver MC, Smith EC, Sher F, Pinello L, Sanjana NE, Shalem O, et al. BCL11A enhancer dissection by Cas9-mediated in situ saturating mutagenesis. Nature. 2015;527:192-7.

52. Sherry S, Ward M-H, Kholodov M, Baker J, Phan L, Smigielski E, et al. dbSNP: the NCBI database of genetic variation. Nucleic Acids Res. 2001:29:308-11.

53. Schaefer KA, Wu W-H, Colgan DF, Tsang SH, Bassuk AG, Mahajan VB. Unexpected mutations after CRISPR-Cas9 editing in vivo. Nat Methods. 2017; 14:547-8.

54. Ihry RJ, Worringer KA, Salick MR, Frias E, Ho D, Theriault $K$, et al. p53 inhibits CRISPR-Cas9 engineering in human pluripotent stem cells. Nat Med. 2018; 24:939-46.

55. Haapaniemi E, Botla S, Persson J, Schmierer B, Taipale J. CRISPR-Cas9 genome editing induces a p53-mediated DNA damage response. Nat Med. 2018:24:927-30.

56. Lareau CA, Clement K, Hsu JY, Pattanayak V, Joung KJ, Aryee MJ, et al. Response to "Unexpected mutations after CRISPR-Cas9 editing in vivo". Nat Methods. 2018;15:238-9.

57. Nutter LM, Heaney JD, Lloyd KK, Murray SA, Seavitt JR, Skarnes WC, et al. Response to "Unexpected mutations after CRISPR-Cas9 editing in vivo". Nat Methods. 2018;15:235-6.

58. Kim S-T, Park J, Kim D, Kim K, Bae S, Schlesner M, et al. Response to "Unexpected mutations after CRISPR-Cas9 editing in vivo". Nat Methods. 2018;15:239-40

59. Wilson CJ, Fennell T, Bothmer A, Maeder ML, Reyon D, Cotta-Ramusino C, et al. Response to "Unexpected mutations after CRISPR-Cas9 editing in vivo". Nat Methods. 2018;15:236-7.

60. Lescarbeau RM, Murray B, Barnes TM, Bermingham N. Response to "Unexpected mutations after CRISPR-Cas9 editing in vivo". Nat Methods. 2018;15:237.

61. Iyer V, Boroviak K, Thomas M, Doe B, Riva L, Ryder E, et al. No unexpected CRISPR-Cas9 off-target activity revealed by trio sequencing of gene-edited mice. PLoS Genet. 2018;14:e1007503.

62. Martins VC, Busch K, Juraeva D, Blum C, Ludwig C, Rasche V, et al. Cell competition is a tumour suppressor mechanism in the thymus. Nature. 2014;509:465-70.

63. Schiroli G, Ferrari S, Conway A, Jacob A, Capo V, Albano L, et al. Preclinical modeling highlights the therapeutic potential of hematopoietic stem cell gene editing for correction of SCID-X1. Sci Transl Med. 2017:9:eaan0820.

64. Tsai SQ. Towards safe therapy for immunodeficiency. Nat Biomed Eng. 2017;1:937.

65. Yang L, Grishin D, Wang G, Aach J, Zhang C-Z, Chari R, et al. Targeted and genome-wide sequencing reveal single nucleotide variations impacting specificity of Cas9 in human stem cells. Nat Commun. 2014;5:5507.

Ready to submit your research? Choose BMC and benefit from:

- fast, convenient online submission

- thorough peer review by experienced researchers in your field

- rapid publication on acceptance

- support for research data, including large and complex data types

- gold Open Access which fosters wider collaboration and increased citations

- maximum visibility for your research: over $100 \mathrm{M}$ website views per year

At $\mathrm{BMC}$, research is always in progress.

Learn more biomedcentral.com/submissions 